\title{
Examination of the Mesoscopic Scale Response of Shock Compressed Heterogeneous Materials Using a Line-Imaging Velocity Interferometer ${ }^{*}$
}

\author{
W. M. Trott, J. N. Castañeda, J. J. O'Hare, M. D. Knudson, L. C. Chhabildas, M. R. Baer, and J. R. Asay \\ Sandia National Laboratories \\ Albuquerque, NM 87185-0834
}

In order to provide detailed response data for validation of three dimensional numerical simulations of heterogeneous materials subjected to impact loading, an optically recording velocity interferometer system (ORVIS) has been adapted to a line-imaging instrument capable of generating precise mesoscopic scale measurements of spatially resolved velocity variations during dynamic deformation. While the use of this diagnostic has been demonstrated on several different classes of heterogeneous materials, studies have focused on pressed, granular sugar as a simulant material for the widely used explosive HMX. Tests on this material address mesoscopic scale thermomechanical effects in the absence of complications due to rapid reactions. For low-density ( 65 percent theoretical maximum density) pressings of sugar, material response has been investigated as a function of both impact velocity and changes in particle size distribution. The amplitude and frequency of observed transverse and longitudinal wave structures are generally consistent with the highly structured mesoscopic response seen in the 3-D simulations. In parallel with instrumental developments, several approaches for detailed analysis of the spatially resolved velocity-time data are being evaluated. The utility of Fast Fourier Transform (FFT) filtering for reduction of certain types of ORVIS image data has been demonstrated.

\section{INTRODUCTION}

Microscopic material descriptions such as grain morphology, internal defects, tensile strength, shear behavior, heat conduction, etc. in large part drive the detailed response of a heterogeneous material (e.g., a pressed granular explosive) to impact loading. Analysis of this detailed response is addressed by the current capabilities for three-dimensional (3-D) numerical simulations. [1] These calculations explore the correlation between microscopic properties and the response of the material at mesoscopic scales, including detailed spatial variations in stress and thermal fields (dispersive behavior). To support the computational efforts, there is a critical need for diagnostic development that can effectively probe the material response at the requisite mesoscopic scale. Characteristic length scales can vary from a few $\mu \mathrm{m}$ (e.g., for PZT materials) to a few mm (e.g., for concrete).
Spatially resolved velocity interferometry provides a very promising diagnostic approach in this area of research. Various ideas for extending conventional "single point" methods to instruments capable of measurements at multiple points or across line segments and surfaces have been actively investigated. [2-6] In 1995 Baumung et al. [7] described a simplified design for a high-resolution, line-imaging interferometer with modest requirements $(\sim 1 \mathrm{~W})$ for the laser source. We have utilized similar approaches in adapting the conventional optically recording velocity interferometer system (ORVIS) configuration [8] to fine-scale, spatially resolved measurements. We are currently using an instrument of this type in wellcontrolled, well-characterized impact loading experiments at a gas gun facility. Initial tests exploring the mesoscopic scale dynamic response of a variety of heterogeneous materials have been discussed elsewhere. [9]

\footnotetext{
Sandia is a multiprogram laboratory operated by Sandia Corporation, a Lockheed Martin Company for the United States Department of Energy under Contract DE-AC04-94AL85000.
} 


\section{DISCLAIMER}

This report was prepared as an account of work sponsored by an agency of the United States Government. Neither the United States Government nor any agency thereof, nor any of their employees, make any warranty, express or implied, or assumes any legal liability or responsibility for the accuracy, completeness, or usefulness of any information, apparatus, product, or process disclosed, or represents that its use would not infringe privately owned rights. Reference herein to any specific commercial product, process, or service by trade name, trademark, manufacturer, or otherwise does not necessarily constitute or imply its endorsement, recommendation, or favoring by the United States Government or any agency thereof. The views and opinions of authors expressed herein do not necessarily state or reflect those of the United States Government or any agency thereof. 


\section{DISCLAIMER}

Portions of this document may be illegible in electronic image products. Images are produced from the best available original document. 
In this paper we briefly describe essential elements of the current line-imaging ORVIS optical design along with a complementary technique for simultaneous measurement of an areal (2-D) velocity map at one point in time. Also discussed are methods for line-imaging ORVIS data reduction. The primary analysis tool is a program that generates spatially resolved velocity-time records by adapting widely utilized "push-pull" VISAR analysis methods. [10] Some advantages and limitations of Fast Fourier Transform (FFT) filtering of the ORVIS image data for this purpose are presented. The mesoscopic scale response of low-density pressings of coarse, granular sugar (a chemically inert simulant for the high explosive, HMX) to shock loading has been examined as a function of both impact velocity and changes in particle size distribution. Experimental results that capture both transverse and longitudinal wave structures at this scale are presented. The results compare favorably to the detailed material response predicted by computational modeling of the low-density sugar at the mesoscale.

\section{EXPERIMENTAL}

Techniques for generating a line-imaging ORVIS assembly are illustrated in Fig. 1. As in the conventional configuration, coherent light from the source laser can be directed through a small hole in a turning mirror and then focused onto the target of interest. Diffusely reflected light from the target is collected and roughly collimated by the focusing lens (L1); the collimated beam reflects off the turning mirror and is reduced in diameter by the down-collimating telescope optics (L2, L3). A 50/50 beamsplitter splits the beam into two equal-intensity components, one of which serves as a reference leg. The second leg passes through a variable-length fused silica cylinder (labeled "Delay Bar" in Fig. 1). This optical component imparts a time delay (proportional to the cylinder length) in the second beam. Observed motion of the interference fringes generated by beam recombination (with mirrors tilted so as to produce a "straight-line" pattern) is directly proportional to target velocity. This motion can be recorded by a high-speed streak camera

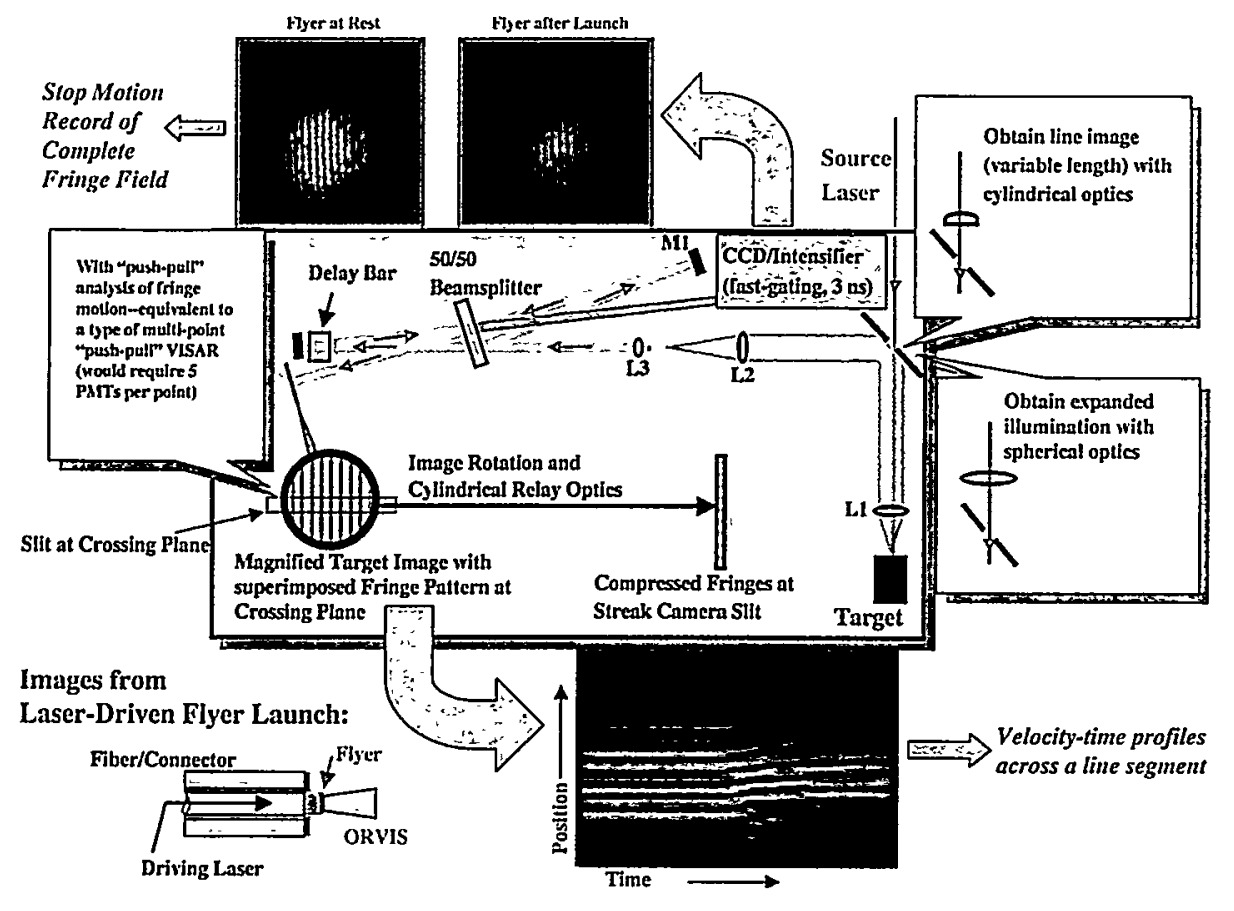

Figure 1. Schematic of experimental configuration for line-imaging ORVIS and stop-motion imaging of full fringe field. 
(image rotation and relay optics may be utilized in this operation, depending on the desired location of the streak camera and the orientation of its entrance slit). The focusing/collection lens (L1) and telescope optics (L2, L3) can be adjusted to produce a magnified image of the target at the recombination plane (i.e., the location of best fringe contrast); in this mode, local variations in target surface velocity are reflected in corresponding local translations of the superimposed fringe field. Facile control of image size and spatial resolution can be achieved via suitable variations in the relative angles and spacing of the interferometer mirrors as well as the spacing and focal lengths of the imaging optics.

As demonstrated by Baumung et al., the target illumination can be confined to a thin line segment by placing a cylindrical lens of suitable focal length in the source laser path ahead of the turning mirror aperture. [7] Alternatively, one can place a spherical lens in this position and expand as desired the illumination area on target. A line image for recording by the streak camera can still be produced in this mode by placing a rectangular aperture of the desired width near the image/recombination plane. This approach requires a suitable combination of source laser power and target reflectivity to ensure sufficient signal at the streak camera; however, it enables a powerful complementary technique that utilizes a redundant recombined beam that propagates off the front side of the beamsplitter (cf. Fig. 1). In probing this beam, a fast-gating ( $<3 \mathrm{~ns})$ intensifier/CCD can be used to capture a stopmotion image of the full fringe field at one point in

(a)

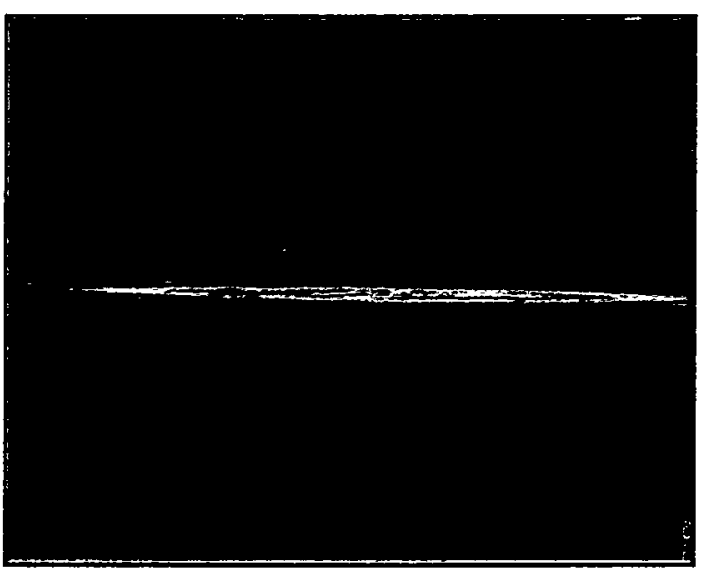

time. In addition to producing data that can be reduced to generate a 2-D map of target surface velocity at one instant, this technique provides an informative diagnostic for the behavior of the lineimaging ORVIS fringe records (e.g., in locating regions of surface curvature that leads to vignetting of signal at the streak camera).

An interesting and useful refinement of the approach to line illumination reported by Baumung et al. [7] involves the removal of $\mathrm{L} 1$ from the source laser beam path and relocation of this element to a position between the turning mirror and L2. In this configuration, established techniques of laser light sheet generation [11] (employing a combination of spherical and cylindrical optics) can be used to tailor independently the width and length of the illumination line segment on target. Line segments of very narrow width $(<150 \mu \mathrm{m})$ can be produced in this manner, as shown in Fig. 2. With this approach, the target illumination optics are completely decoupled from the collection optics in general and the focal length of L1, in particular. An additional advantage of this method is the elimination of source laser light (i.e., backreflected from L1) in the fringe signal reaching the streak camera.

A compact version of the line-imaging ORVIS has been fielded at the Explosive Components Facility (ECF) gas gun at Sandia National Laboratories. This system combines the interferometer optics, laser source and streak camera/intensifier/CCD detector on a single ' ' $\times 6^{\prime}$ ' optical breadboard. Coupling of this diagnostic to the test samples required design and assembly of a

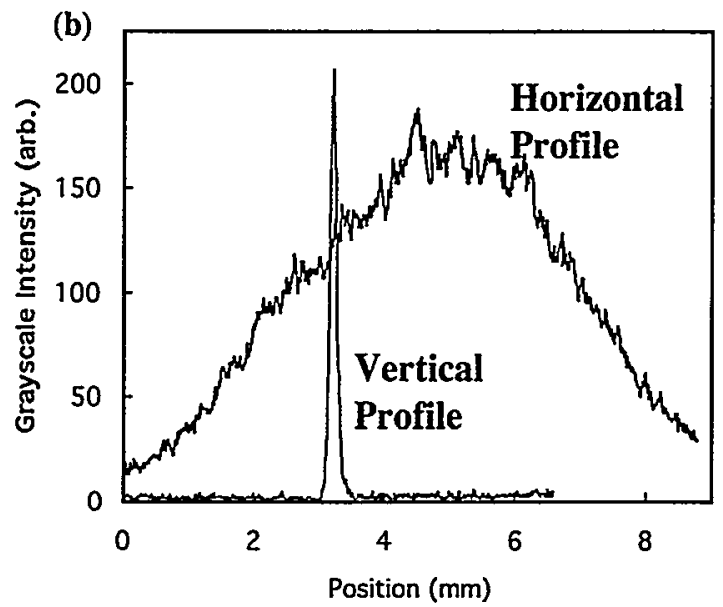

Figure 2. Beam profile data for line segment target illumination using laser light sheet optics: (a) CCD camera image of line illumination; (b) intensity profiles illustrating narrow width of line. 
complex optical interface that could accommodate the 11-meter path to the gas gun target chamber. Targets that allow for simultaneous line-imaging ORVIS and standard dual-delay-leg, "push-pull" VISAR measurements on a variety of materials have been designed and fabricated. Simultaneous use of both techniques permits direct comparison of spatially resolved vs. single spot data.

\section{DATA REDUCTION METHODS}

To exploit the information associated with each recorded fringe in line-imaging ORVIS data, it is necessary to develop a suitable method for image analysis. Baumung et al. [7] described an approach (analogous to reduction of VISAR interferograms) that extracts the intensity modulation data along lines parallel to the time axis of the streak camera record. The simplest implementation of this reduction method generates components that are, in effect, quadrature coded using intensity "lineouts" along the center of a fringe and at $1 / 4$ the distance to the center of a neighboring fringe.

As described previously, adapting the "push-pull" VISAR analysis methods to reduce the ORVIS fringe records yields a more robust approach. [9] In this technique, "lineouts" are recorded at positions corresponding to the fringe center as well as at $1 / 4$, $1 / 2$, and $3 / 4$ the distance to the center of a neighboring fringe. These data can be treated in the same manner as that recorded by four PMT channels in a "push-pull" VISAR. [10] This approach is shown schematically in Fig. 3. It is important to note that it is not a requirement to register the lineout data set for each single-line analysis with a fringe center. Considerably finer resolution of various small-scale velocity perturbations can be achieved by extending

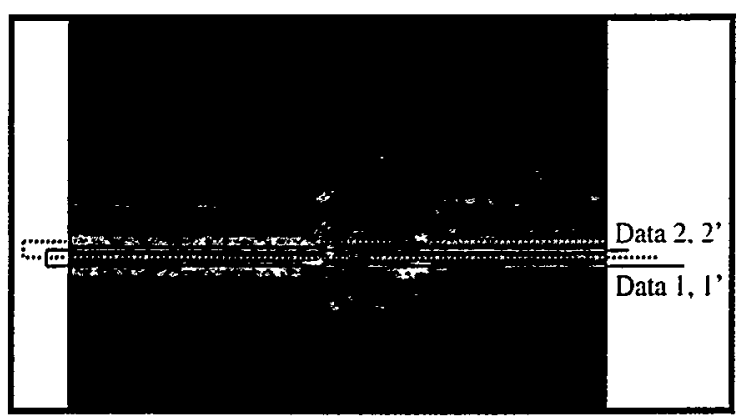

Figure 3. Illustration of intensity "lineout" positions for input to "push-pull" analysis routine. the analysis to positions between fringes as well. This "rolling push-pull" approach has been used in reduction of the experiments described below. An analogous routine can be used to generate an areal target surface velocity map (at one point in time) from stop-motion images of the fringe field.

A computer software package that integrates the "rolling push-pull" technique with the necessary image processing methods and an existing VISAR data analysis platform has been developed. Briefly summarized, the typical data reduction procedure is as follows: $\{a\}$ conversion of image data to standard TIFF format (if necessary), $\{b\}$ image rotation to level the fringes horizontally (if necessary), \{c\} definition of a region of interest (ROI) on the image data for further processing, (d) evaluation of average fringe spacing in a region of the image corresponding to no target motion, $\{\mathrm{e}\}$ image expansion and interpolation to adjust the average fringe spacing to a pixel value evenly divisible by four (a step that allows each data set to be registered to either a fringe center or positions $1 / 4,1 / 2$, or $3 / 4$ the distance to the center of an adjacent fringe), $\{\mathrm{f}\}$ evaluation of intensity variations along the time axis for positions corresponding to each 1/4-fringe, $\{\mathrm{g}\}$ conversion of these intensity lineouts to a series of equivalent "push-pull" data sets, $\{h\}$ export of data to the VISAR analysis package to extract velocitytime records corresponding to each 1/4-fringe position, and \{i\} export of velocity-time profiles to a 3-D plotting package. The software package also includes an optional regression solver to assist in evaluation of fringe spacing for fringes of widely disparate intensities as well as various filters for smoothing the intensity-time data.

In tests producing relatively modest fringe displacement, it is necessary to account for systematic drift in the detector sweep. This drift pattern can be detected in baseline traces (i.e., fringes obtained from a static target). The analysis software provides the option of reducing a baseline record in parallel with a test record, largely accounting for spurious contributions to the velocitytime profiles in the process.

\section{APPLICATION TO HETEROGENEOUS MATERIALS}

Line-imaging ORVIS has been used to investigate the mesoscopic scale dynamic response of several different classes of heterogeneous materials under 
gas-gun-driven impact loading, including foam and glass-reinforced polyester. The preponderance of our efforts has focused on the investigation of pressed, granular sugar as a simulant material for the explosive HMX. The utility of this candidate simulant has been previously described. [12] Chemically inert to shock loading at fairly high pressures, granulated sugar is reported to be similar in particle size distribution to typical batches of "coarse" HMX used in various applications. Tests on this material can address mesoscopic scale thermomechanical effects in the absence of complications due to rapid reactions. Our tests are intended to provide experimental data for direct comparison and validation of 3-D numerical simulations focusing on the thermomechanical response. [1] In addition, the line-imaging ORVIS results can be directly compared to the substantial data base developed from magnetic gauge ("continuum") experiments by Sheffield et al. [12]

Accordingly, simultaneous ORVIS and standard VISAR measurements have been made on the wave transmitted by 4-mm-thick pressed sugar samples in a gas gun target design very similar to that used in the previous magnetic gauge studies; i.e., Kel-F impacting sugar pressed to $65 \%$ theoretical maximum density (TMD) in a Kel-F target cup. Earlier versions of the target design did not allow for spatially overlapped VISAR and line-imaging ORVIS. [9] Spatial overlap of these diagnostics is, however, accommodated by the current target design, as shown in Fig. 4.

In tests conducted thus far, impact velocities ranging from $0.32-0.68 \mathrm{~km}-\mathrm{s}^{-1}$ have been used. Initial results from both VISAR and line-imaging
ORVIS are consistent with the systematically varying dispersive behavior of the wave profiles seen by Sheffield et al. [12] This includes comparable measured shock and particle velocities as well as very similar rise times in the transmitted wave. In particular, the measured rise times decrease from $700 \mathrm{~ns}$ to approximately $200 \mathrm{~ns}$ over the above range of impact velocities. Mesoscopic scale velocity variations (both transverse and longitudinal wave structures) are clearly evident in the spatially resolved ORVIS data.

Variations in the observed amplitude and frequency of oscillations in the wave profile vs. position provide insight at the mesoscale that should be helpful in defining appropriate material descriptions and interface conditions for detailed computational modeling. [1] As a first step toward a meaningful comparison between the experiments and the 3-D simulations, we have attempted to characterize the particle size distribution for the sugar used in our tests. In particular we sieved our lot of coarse, granulated sugar under two conditions: [1] as received, and [2] after pressing to $65 \% \mathrm{TMD}$ (into the Kel-F target cup) followed by release. The results of this operation for the latter case are shown in Fig. 5. In both cases the largest weight fraction was found in a very coarse "bin" of material (250$425 \mu \mathrm{m}$ diameter). In the pressed and released sample, significantly higher weight fractions were observed in the fine-grain ( $<150 \mu \mathrm{m})$ "bins." Hence, the effect of grain crushing is significant even at the low pressing density used in this study.

For computational modeling of the response of the low-density pressing of sugar under the impact conditions examined here, the measured particle size

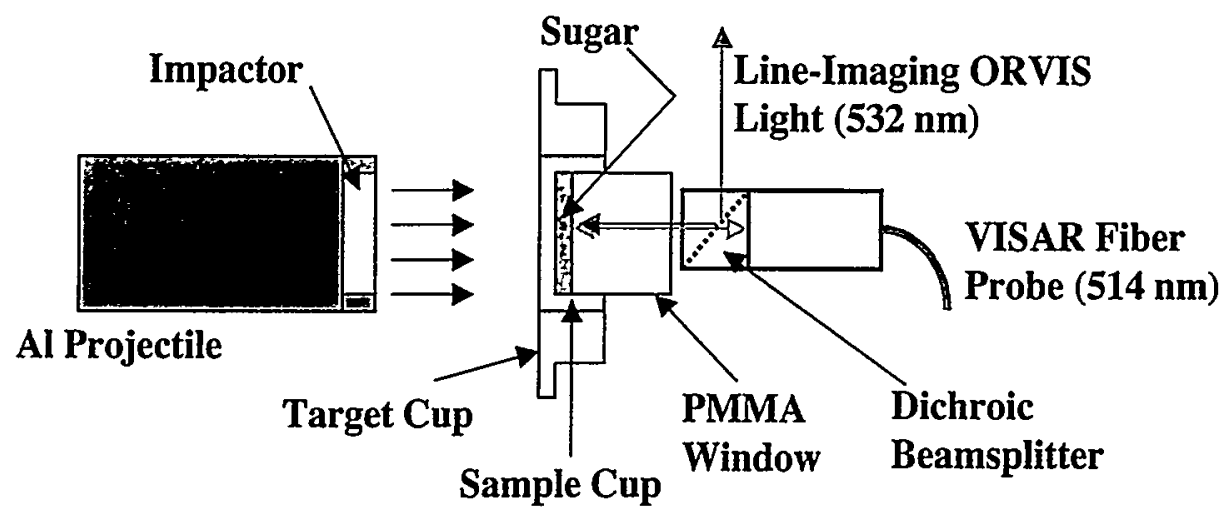

Figure 4. Schematic diagram of gas gun target design for measurements on low-density sugar samples. Use of the dichroic beamsplitter enables spatially overlapped point VISAR and line ORVIS diagnostics. 


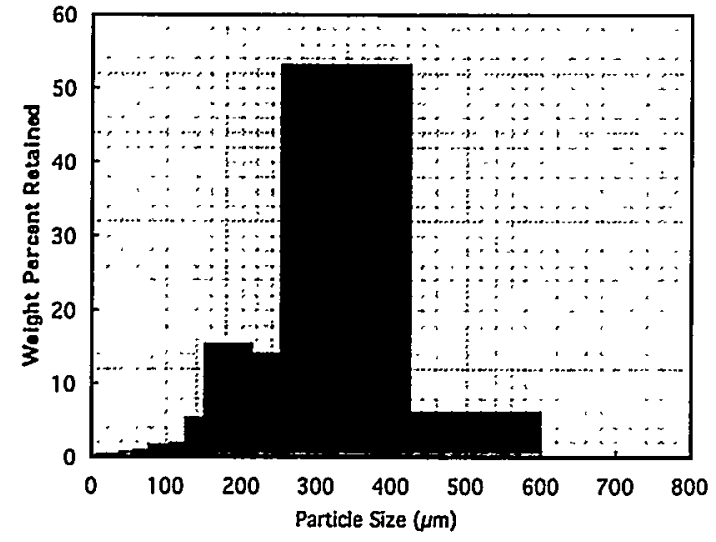

Figure 5. Particle size distribution determined for granulated sugar (pressed and released).

distribution of the pressed and released sample (expressed in terms of weight fraction) was converted to an equivalent number density distribution. This distribution was incorporated into packing algorithms described previously [1] in order to generate a random ensemble of sugar crystals consistent with that used in the experiments. Periodic lateral boundary conditions were placed on the computational domain. The wave field was transmitted through a buffer layer of Kapton (225 $\mu \mathrm{m}$ thick as employed in the experiments) and into the PMMA material used as an interferometer window. Particle velocity was recorded at approximately 50 "tracer" points along a line segment located at the Kapton/PMMA interface.

In the simulations, rapid deformation occurs at material contact points in the sugar. Both stress and temperature fields display a substantial degree of large amplitude fluctuations. These fluctuating fields arise from the effects of shocks interacting with individual material surfaces and multiple crystal interactions. Figure 6 displays a time sequence of temperature fields along a midplane cross-section of the low-density sugar bed. Times were chosen to illustrate interesting dispersive wave interaction events at the sugar/Kapton and Kapton/PMMA interfaces. Of particular interest is the material penetration event at the location marked by an arrow in Fig. 6. This effect is also clearly evident in the stress field contours. Ideally, it would be useful to acquire particle velocity measurements without the attenuating influence of a buffer layer. In practice, however, we have found that substantial loss in the reflected light intensity for both point VISAR and line-imaging ORVIS occurs upon wave arrival at the buffer/PMMA interface when buffer thicknesses $<150 \mu \mathrm{m}$ are used. This observation is consistent with the crystal penetration depth seen in the computations.

Fortunately, the calculated particle velocities retain a significant degree of amplitude fluctuation even after transmission through a 225 - $\mu$ m-thick buffer, as shown in Fig. 7. The 3-D plot of velocitytime profiles at the Kapton/PMMA interface

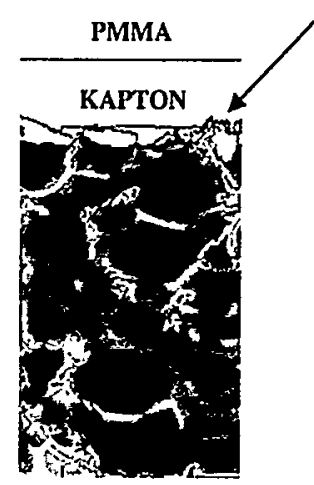

(a)

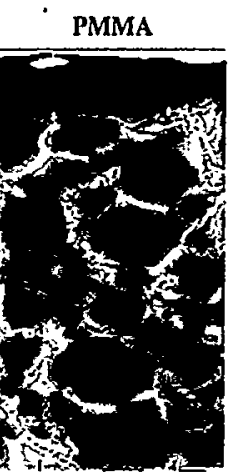

(b)

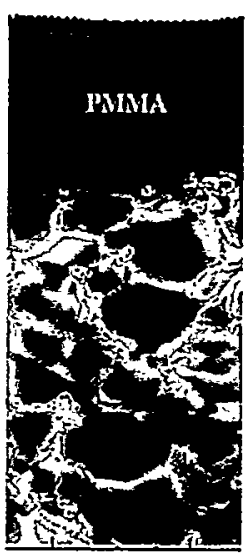

(c)

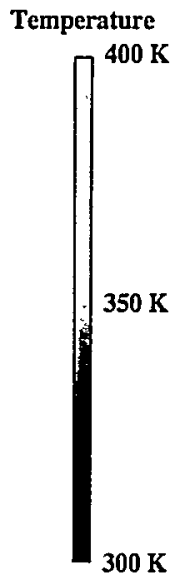

$300 \mathrm{~K}$

Figure 6. Time sequence of temperature field maps from numerical simulation of low-density sugar under impact loading: (a) initial interaction with Kapton buffer layer; (b) wave approaching PMMA; (c) crystal penetration into Kapton layer. 

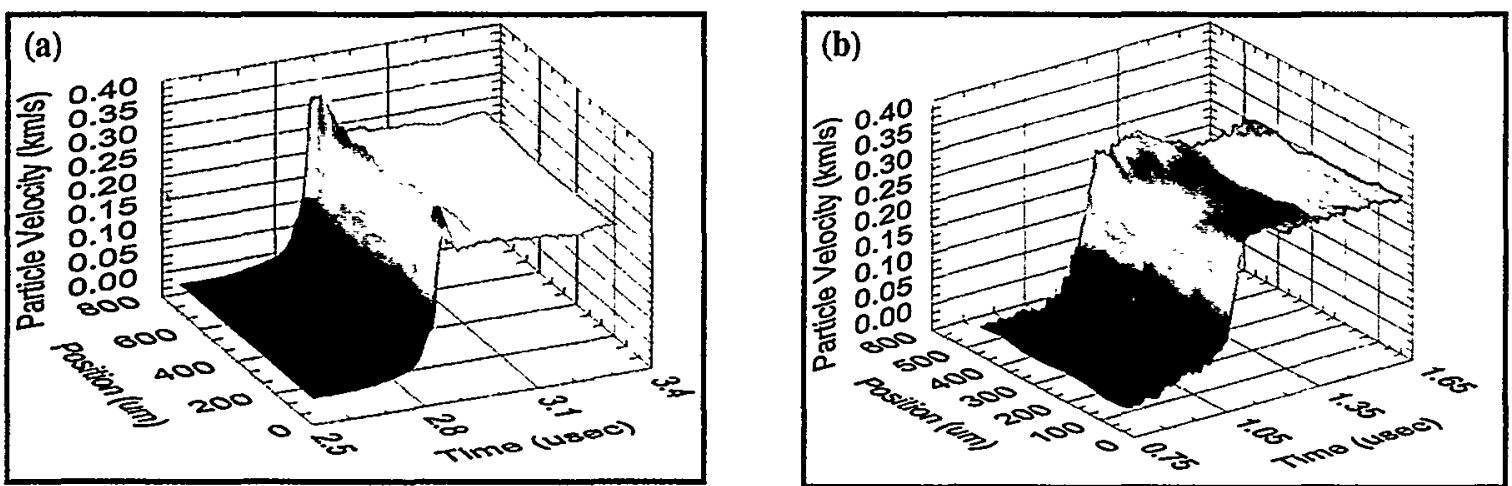

Figure 7. Comparison of (a) results from CTH calculation and (b) spatially resolved velocity-time profiles obtained using line-imaging ORVIS; $0.5 \mathrm{~km}-\mathrm{s}^{-1}$ impact velocity.

displays complex transverse mode structure in addition to the 110 -ns rise time in the wave. A representative plot of the spatially resolved velocitytime field observed by line-imaging ORVIS is also presented in Fig. 7. Considering important differences in the material geometry employed in experiments vs. the simulations (the complexity of the computations precluded the use of a sugar bed as thick as $4 \mathrm{~mm}$ ), the agreement is reasonably good. Both experiment and computations exhibit early peaks in the particle velocity (with significant transverse wave structure), followed by late-time velocities near $0.25 \mathrm{~km}-\mathrm{s}^{-1}$.

It is instructive to average the line-imaging ORVIS data over the entire line segment and compare it to the similarly averaged calculated response. This comparison is illustrated in Fig. 8. Also shown is the

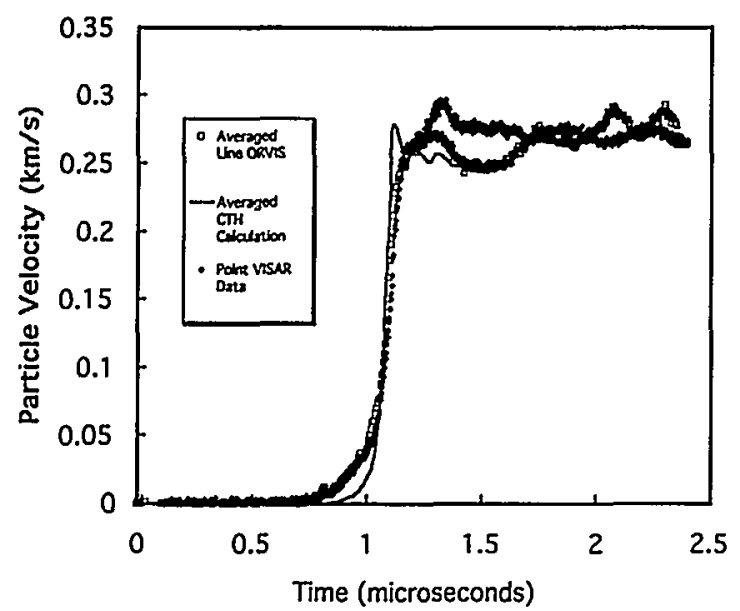

Figure 8. Comparison of point VISAR and averaged line-imaging ORVIS data to averaged response from numerical simulation. point VISAR record obtained in this test (in this case, the VISAR and ORVIS data were acquired at different locations on the target). To aid in the comparison, the traces have been shifted in time to overlap at the $0.1 \mathrm{~km}-\mathrm{s}^{-1}$ level. The VISAR and ORVIS data are closely correlated in term of rise time but significant differences in wave structure are evident on the "plateau." Both sets of data indicate more dispersion than the calculated result. We attribute much of this effect to wave propagation through a reduced length of sugar in the simulations (we have not yet determined the length required for steady wave behavior).

Perhaps the most promising aspect of these preliminary comparisons of experiment and theory is that the length scale and spatial resolution afforded by line-imaging ORVIS appear to address the relevant scale for wave fluctuations seen in the numerical simulations. Much work remains to be done, however, in order to obtain a sufficient data base to test many of the critical properties of the computational model. Aside from the obvious need for a closer match in material geometry, the variations in the VISAR and ORVIS data in Fig. 8 demonstrate the statistical nature of the measured response of a heterogeneous sugar sample. For each condition of impact velocity, density and particle size distribution, a suitable collection of spatially resolved velocity-time profiles must be acquired in order to sample a sufficient number of crystal/void ensembles to characterize the system properly.

Particle size distribution is a critical parameter that can easily be varied. Initial results with tailored distributions of low-density sugar indicate substantial differences in response to impact loading. 

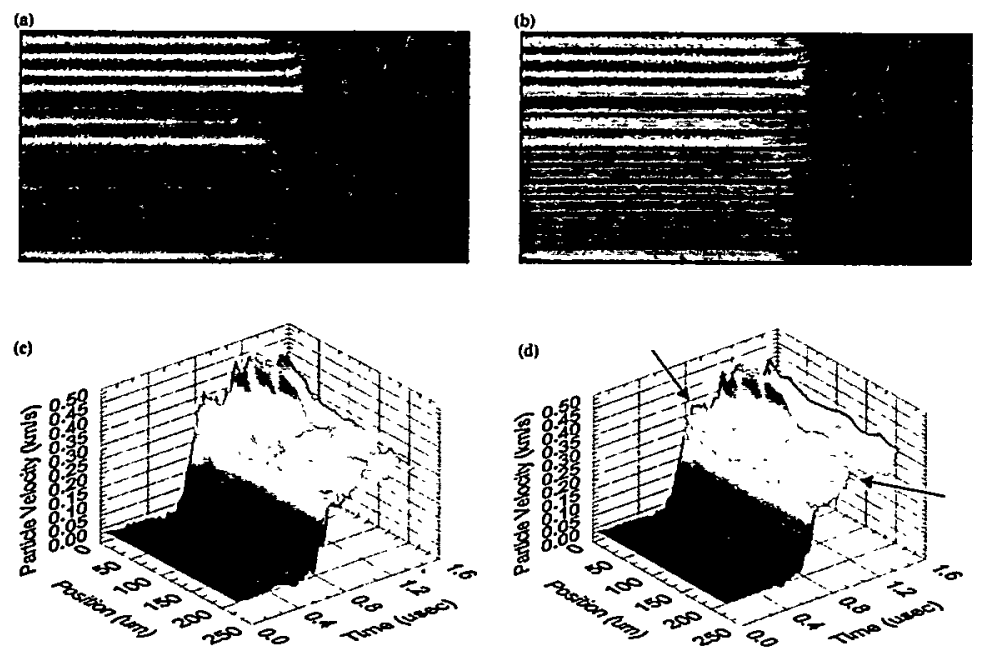

Figure 9. Application of FFT filter to line-imaging ORVIS data: (a) unfiltered image; (b) FFT-filtered image; (c) velocity profile from unfiltered image; (d) velocity profile from filtered image.

For these tests, we prepared two "sieve cut" samples for examination under impact loading: [1] a coarse cut sample using the predominant $250-425 \mu \mathrm{m}$ fraction, and [2] a relatively fine-grain sample using 150-212 $\mu \mathrm{m}$ particles. As expected, significantly higher force was required to press the coarse-grain sample to $65 \% \mathrm{TMD}$ as a result of its restricted ability to pack uniformly. A substantially larger extent of grain fracturing is very likely in this case. The two pressed samples were examined upon impact at $0.42 \mathrm{~km}-\mathrm{s}^{-1}$ using simultaneous lineimaging ORVIS and VISAR. Both diagnostics indicate more dispersive wave behavior in the coarse grain sample. In particular, the ORVIS and VISAR wave profiles in this case exhibit significantly longer rise times as well as generally higher amplitude oscillations. Results of this type should provide an informative test of the accuracy of mesoscopic scale numerical simulations.

We are also exploring advanced methods for processing and analyzing both experimental data and computational results. In certain cases, a Fast Fourier Transform (FFT) filter can be applied to the data with good effect. As shown in Fig. 9, this method can be used to obtain better fringe definition and contrast. This is particularly useful when the signal intensity is very low and contaminated with random noise. It is well known, however, that the bases of Fourier analysis are severely limited in approximating sharp spikes and discontinuities. As a result, important data fluctuations can be suppressed upon application of the FFT filter. Two possible examples of this effect are marked with arrows in Fig. 9d. Wavelet transforms provide a more specific approach that allows different frequency components to be analyzed with a resolution matched to scale. [13] We are currently investigating the utility of wavelets for processing results in our applications.

\section{REFERENCES}

1. M.R. Baer, in Shock Compression of Condensed Matter-1999 (M. Furnish, et al. eds), pp. 27-33.

2. S. Gidon and G. Behar, Appl. Optics 25 (1986) 1429.

3. A.R. Mathews, R.H. Warnes, W.F. Hemsing, and G.R. Whittemore, SPIE Proc. No. 1346, 122.

4. W.F. Hemsing, A.R. Mathews, R.H. Warnes, and G.R. Whittemore, SPIE Proc. No. 1346, 133.

5. A.R. Mathews, R.M. Boat, W.F. Hemsing, R.H. Warnes, and G.R. Whittemore, in Shock Compression of Condensed Matter-1991 (S. Schmidt et al., eds.), pp. 759-762.

6. L.M. Barker, in Shock Compression of Condensed Matter-1999 (M. Furnish, et al. eds), pp. 999-1002.

7. K. Baumung, J. Singer, S.V. Razorenov, and A.V. Utkin, in Shock Compression of Condensed Matter-1995 (S. Schmidt and W. Tao, eds.), pp. 1015-1018.

8. D. D. Bloomquist and S.A. Sheffield, J. Appl. Phys. 54 (1983) 1717.

9. W.M. Trott, M.D. Knudson, L.C. Chhabildas, and J.R. Asay, in Shock Compression of Condensed Matter-1999 (M. Furnish, et al. eds.), pp. 993-998.

10. W.F. Hemsing, Rev. Sci. Instrum. 50 (1979), 73.

11. G. Diemunsch and J.P. Prenel, Optics and Laser Technology 19 (1987), 141.

12. S.A. Sheffield, R.L. Gustavsen, and R.R. Alcon, in Shock Compression of Condensed Matter-1997, (S. Schmidt, et al., eds.), pp. 575-578.

13. M. Farge, Ann. Rev. Fluid Mech. 24 (1992) 395. 